\title{
Tailoring Thermal Expansion Coefficient of Transition Metal Dichalcogenides via Alloy Engineering
}

\author{
Xuan $\mathrm{Hu}^{1}$, Amirhossein Behranginia ${ }^{2}$, Serdar Öğ̈̈t ${ }^{1}$, Amin Salehi $^{2}$, Robert F. Klie ${ }^{1}$ \\ ${ }^{1}$ Department of Physics, University of Illinois at Chicago, Chicago, Illinois, United States \\ 2. Mechanical Engineering Department, University of Illinois at Chicago, Chicago, Illinois, United States
}

Transition metal dichalcogenides (TMDs) and 2-dim materials beyond graphene have shown excellent potential for future electronics [1-2]. Controlling the heat flow across a hetero-structure will be crucial to developing high-speed electronic devices based on 2-dim materials. We have recently shown that the thermal expansion coefficient (TEC) dramatically increases in 2-dim materials when the thickness of the material shrinks from bulk to a few monolayers [3]. Therefore, the TEC mismatch of 2-dim materials becomes an additional concern in designing electronic nano-devices. More specifically, we need to develop methods that enable us to control and tailor the TEC of TMDs through alloying or defect engineering.

In this contribution, we will employ transition metal alloying in TMDs to tune the TEC of monolayer $\mathrm{Mo}_{1-\mathrm{x}} \mathrm{W}_{\mathrm{x}} \mathrm{S}_{2}$ and study the interplay between thermal expansion and local defects using a combination of the scanning transmission electron microscope (STEM), electron energy loss spectroscopy (EELS) and first-principles DFT calculations. More specifically, we will measure the thermal expansion coefficient based on the plasmon energy shift as a function of temperature and combine this with first-principles modeling of the low-loss EELS signals [3]. Figure 1A shows an example of typical EELS plasmon peak shift as a function of temperature for a monolayer $\mathrm{WSe}_{2}$. Using DFT calculations in the random phase approximation (RPA) we model the plasmon peak shift as a function of lattice expansion (Figure 1B). Combining the experimental and modeling data, we can now predict the TEC for $\mathrm{WSe}_{2}$ [3].

Using this approach, we have determined the TEC of monolayer $\mathrm{MoS}_{2}$ and $\mathrm{WS}_{2}$ and found a significant mismatch between the two materials. To explore the influence of alloy engineering on the TEC, freestanding $\mathrm{Mo}_{0.7} \mathrm{~W}_{0.3} \mathrm{~S}_{2} 2$-dim materials are prepared. Figure 2A shows the low angle annular bright field (LAADF) image of a few layer $\mathrm{Mo}_{0.7} \mathrm{~W}_{0.3} \mathrm{~S}_{2}$ at $\mathrm{T}=250{ }^{\circ} \mathrm{C}$. The relative thickness is calculated from the low-loss EELS showing that the whole area has a constant thickness. From this Z-contrast image, we find that the bright spots in Figure 2A consist of heavier atoms (W atoms), while the darker spots present Mo atoms. Figure 2A shows that most of these Mo and $\mathrm{W}$ atoms are randomly distributed, while we also found a few small clusters of $\mathrm{WS}_{2}$ and $\mathrm{MoS}_{2}$ as well. Figure $2 \mathrm{~B}$ shows the plasmon peak map from the low-loss EELS signals at $\mathrm{T}=250{ }^{\circ} \mathrm{C}$. It is interesting to note that the plasmon peak position changes across the field of view although the specimen thickness is constant in this region. Compare Figure 2B with Figure 2A, we can find that the region with a higher plasmon peak position corresponds with the presence of $\mathrm{WS}_{2}$ clusters in the $\mathrm{MoS}_{2}$ matrix, which is consistent with the fact that the plasmon peak position for pure $\mathrm{WS}_{2}$ is higher than for pure $\mathrm{MoS}_{2}$ [3]. Measurements from the same region at $\mathrm{T}=150{ }^{\circ} \mathrm{C}$ and the line scans of plasmon peak positions at different temperatures are shown in Figure $2 \mathrm{C}$. We find an overall increase in plasmon peak positions as temperature decreases and note that the relative plasmon shift is $-2.3 \mathrm{meV} / \mathrm{K}$, which corresponds to a linear combination of the plasmon peak shifts from pure $\mathrm{MoS}_{2}$ and $\mathrm{WS}_{2}$. 
In this contribution, we will compare the TEC of the alloyed $\mathrm{Mo}_{0.7} \mathrm{~W}_{0.3} \mathrm{~S}_{2}$ monolayer with that of $\mathrm{MoS}_{2} / \mathrm{WS}_{2}$ lateral heterointerfaces and explore the effects of strain or point defects on the local TEC using a combination of STEM imaging, EEL spectroscopy and DFT modeling [4].

References:

[1] B. Standley et al, Nano Lett. 8 (2008), p. 3345.

[2] W. Han et al, Nat Nano 9 (2014), p. 794.

[3] X. Hu et al, Phys. Rev. Lett. 120 (2018), p. 055902

[4] This work was supported by the National Science Foundation (EFMA-1542864 (EFRI)).
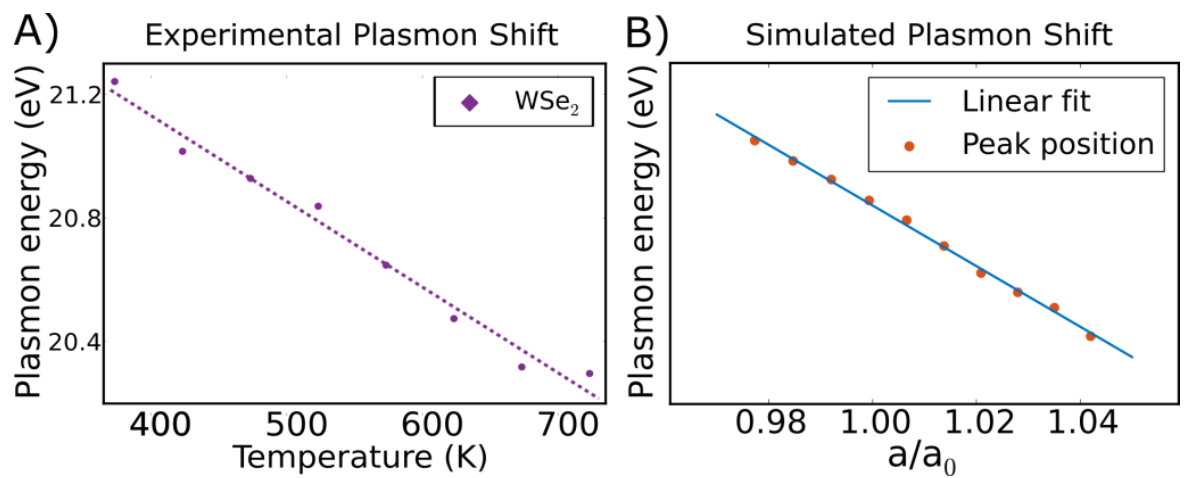

Figure 1. A) An example of the experimental plasmon peak shift as a function of temperature for a monolayer $\mathrm{WSe}_{2}$ nanoflake. B) The simulated plasmon peak shift as a function of lattice constant for the same material.
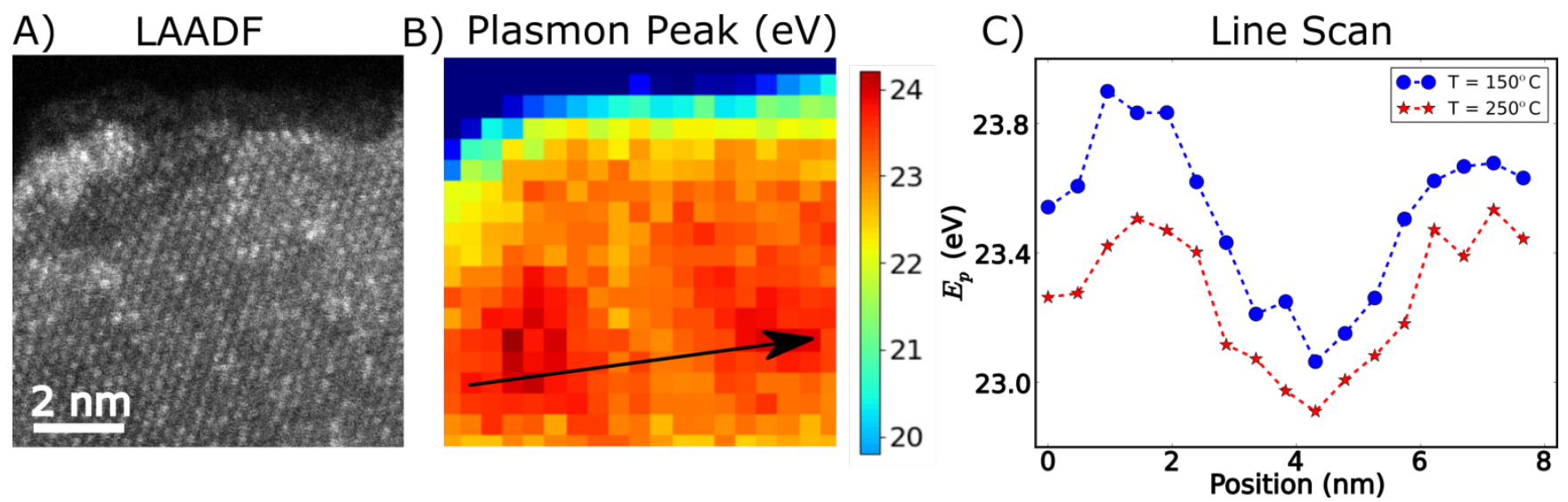

Figure 2. A) LAADF image of a $\mathrm{Mo}_{0.7} \mathrm{~W}_{0.3} \mathrm{~S}_{2}$ nanoflake at $\mathrm{T}=250{ }^{\circ} \mathrm{C}$. B) The plasmon peak map in the same area. C) Line scans of plasmon peaks along the direction of the black arrow in $\mathrm{B}$ ) at $\mathrm{T}=150{ }^{\circ} \mathrm{C}$ (blue line) and $250^{\circ} \mathrm{C}$ (red line). 